\title{
OP48
}

\section{ONE YEAR OF USE OF A LIGHT DOSIMETER}

\author{
Dominique Dumortier et al.
}

DOI 10.25039/x46.2019.OP48

from

CIE x046:2019

\author{
Proceedings \\ of the \\ 29th CIE SESSION \\ Washington D.C., USA, June 14 - 22, 2019 \\ (DOI 10.25039/×46.2019)
}

The paper has been presented at the 29th CIE Session, Washington D.C., USA, June 14-22, 2019. It has not been peer-reviewed by CIE.

(C) CIE 2019

All rights reserved. Unless otherwise specified, no part of this publication may be reproduced or utilized in any form or by any means, electronic or mechanical, including photocopying and microfilm, without permission in writing from CIE Central Bureau at the address below. Any mention of organizations or products does not imply endorsement by the CIE.

This paper is made available open access for individual use. However, in all other cases all rights are reserved unless explicit permission is sought from and given by the CIE.

CIE Central Bureau

Babenbergerstrasse 9

A-1010 Vienna

Austria

Tel.: +4317143187

e-mail: ciecb@cie.co.at

www.cie.co.at 


\title{
ONE YEAR OF USE OF A LIGHT DOSIMETER
}

\author{
Dumortier, D. ${ }^{1}$, Galabertier, P. ${ }^{2}$, Giuly, J. ${ }^{3}$, Rousseau, V. ${ }^{4}$ \\ 1 University of Lyon, ENTPE, LGCB, Vaulx-en-Velin, FRANCE \\ 2 STELC-DREAL Grand Est, Strasbourg, FRANCE \\ ${ }^{3}$ DGALN/DHUP/QC/QC2, MTES, La Défense, FRANCE \\ ${ }^{4}$ VNF/DT Nord-Pas de Calais/SMO/EGT3, Lille, FRANCE
}

dominique.dumortier@entpe.fr

DOI $10.25039 / \times 46.2019 .0 P 48$

\begin{abstract}
We have used a light dosimeter during an entire year. Our objectives were to (1) test its routine use, (2) measure across all seasons, our "real life" exposure to light, as well as our day and night activity, and (3) characterize their variations. The dosimeter was the "LightWatcher" (LW) developed for the EUClock project. It measures UV, Blue, Green, Red and Infrared spectral irradiances as well as activity and temperature. We wore it on an eyeglass frame during the day and on the wrist during the night. We logged our activities. In this paper, we describe the LW sensors and qualify their measurements. We describe how the data was processed. We illustrate some of the first results by showing the difference in light exposure between working days and NOT workings days. We provide feedback on LW use and suggest improvements.
\end{abstract}

Keywords: Dosimeter, Light exposure, Light spectrum, Activity, Season, Sleep

\section{Introduction}

There is now strong scientific evidence that light influences our health and well-being. This happens through the Intrinsically Photosensitive Retinal Ganglion Cells (ipRGCs) (Brainard et al., 2001). These cells connect to a number of so-called non-visual brain structures involved in biological rhythms, sleep and wakefulness, mood, memory, and cognition (Lucas et al., 2014). ipRGCs are directly involved in the effect of light (light therapy), in the treatment of circadian sleep-wake disorders (Auger et al., 2015). They are also involved in the negative effects of light. The deleterious effect of exposure to blue-rich LED screens before bedtime on the quality of sleep and cognition has been shown (Chang et al., 2015), and could be implicated in the growing prevalence of sleep debt and circadian misalignment in industrialized countries, particularly among adolescents and young adults (Czeisler, 2013).

This evidence is based mostly on laboratory studies exposing subjects to specific light and measuring their physiological reaction. Aside from laboratory studies, other studies have looked at our real life exposure to light and its influence on circadian rhythms, noticeably the sleep/wake rhythm. However, for practical reasons, their investigation had to be limited to one or two weeks at specific time(s) of the year (Eastman, 1990; Cole et al., 1995; Scheuermaier et al., 2010). So their characterization of subjects' activity patterns and exposure to light was only representative of these specific times in the year.

Human circadian rhythms are not only diurnal but also seasonal (CIE, 2001). Our activities vary between weekdays and weekends or vacations. Our exposure to daylight indoor or outdoor depends on daylight availability and the type of our activities. These seasonal variations in light exposure may explain seasonal sleep/wake patterns. In order to fully understand the influence of light on our health, our exposure to light and our activity should be characterized over the entire year to take into account seasonal variations. Measuring "in real life" throughout the year, activity and light has become possible because the appropriate technology is now available.

Actimetry has been commonly used to assess the quality of sleep and its position within the 24 hours (early or late in circadian rhythms sleep-wake disorders), or estimate energy expenditure. Many devices exist on the market and are in the form of bracelets worn on the wrist. Some manufacturers have added to their device a measure of "photopic" and/or "RGB" light exposure. However, as measurements are performed at the wrist level, they are not representative of the 
quantity and quality of light received on the retina. In addition, these devices can easily be covered by sleeves. Therefore, actimeters cannot be extrapolated and used to study the impact of light on biological rhythms and sleep.

Prototypes of devices designed to measure light in the plane of the eye have been produced by research laboratories: the ETH Zurich's "LuxBlick" (Hubalek, 2007), the "Daysimeter" of the Rensselaer Polytechnic Institute in Troy, NY-USA (Bierman et al., 2005) and the "LightWatcher" developed in the framework of the European project EUClock (OT, 2016). The "LuxBlick" and the "Daysimeter" measures light producing photopic and melanopic (ipRGCs) illuminances. However, they do not measure activity. The "LightWatcher" measures light producing spectral irradiances on 5 spectral domains (UV, B, G, R and IR). It does measure activity. In addition, it measures ambient temperature. It is available from the Austrian company "Object-Tracker".

The "LightWatcher" is the only miniaturized ambulatory measurement system capable of tracking our exposure to the light spectrum (UV, visible, IR) and our motor activity, day and night. Since we had tested the first prototypes during the EUClock project and since these tests had shown that the device was reliable, we decided to use a "LightWatcher" for an entire year.

Our objectives were to:

- test its practical use over a long period,

- collect one year of light exposure as well as day and night activity,

- characterize the data collected (variations with activity, diurnal and seasonal patterns...),

- study whether the data collected could be used to qualify the activity of the user and his/her environment.

The "LightWatcher" device is shown in figure 1. It has the size of a USB key. We wore it day and night for an entire year. During the day, it was worn on an eyeglass frame in the plane of the right eye. At bedtime, once ready to sleep, it was inserted in a band and worn on the wrist until leaving the bed. It was not used during showers and during data retrieval. Every 5 days, in the evening, at a time of little activity and low exposure to light (watching TV), it was connected to a computer via USB, its data was transferred and it was set to full charge. The whole operation took on average an hour and a half.
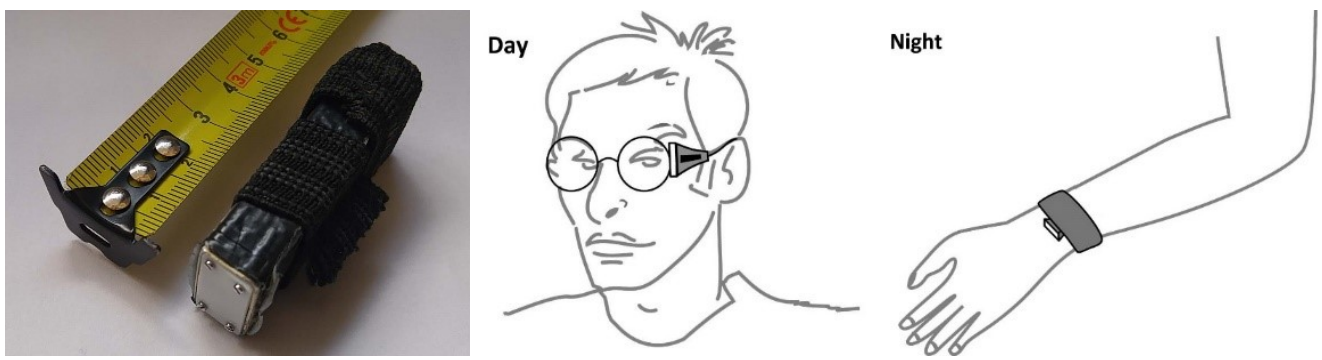

Figure 1 - The light dosimeter was worn on an eyeglass frame during the day and inside a band wrist during the night

All the data has been collected and processed but the analysis is still in progress. In the following, we describe the "LightWatcher" device (called LW for short), the data which was collected and how it was collected, then we provide a few examples of the ongoing analysis.

\section{Data collected}

\subsection{Activity log}

To help in understanding the measurements, we recorded our activities in a diary. An example is given in figure 2. It starts with the weekday name, followed by a numeral indicating whether this is a day of work (1) or not (0) and by the date using the ISO 8601 format, it ends with the activity description. Activities begin with specific keywords: "Go to bed", "Wake up", "Shower", "Drive to...", "Work in...", "Dinner in...", "Watch TV". The qualifier "outside" was added to keywords like "Walk..." or "Work..." when these activities were done outdoor. These keywords 
are used in the analysis to identify the time spent on a given activity and isolate the measurements corresponding to this activity. Every day was documented with 10 to 20 activities. Over the 365 days, a total of 6337 activities were recorded.

Weekday
Thursday
Thursday
Thursday
Thursday
Thursday
Thursday
Thursday
Thursday
Thursday
Thursday
Thursday
Thursday
Thursday
Thursday
Thursday
Thursday
Thursday

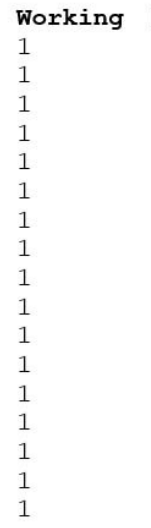

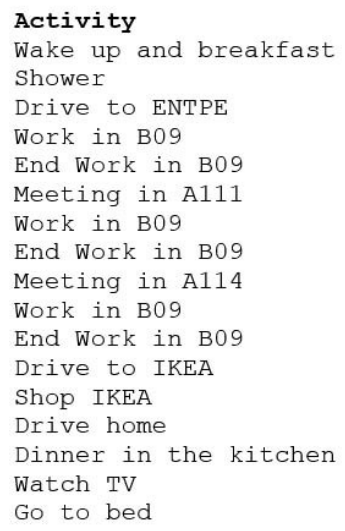

Figure 2 - Sample of the activity log

\subsection{LightWatcher measurements}

\subsubsection{Light measurements}

The LW measures light with 5 photodiodes: UV (from 280 to $370 \mathrm{~nm}$, FWHM of $60 \mathrm{~nm}$ ), Blue (from 380 to $540 \mathrm{~nm}$, FWHM of $80 \mathrm{~nm}$ ), Green (from 460 to $620 \mathrm{~nm}$, FWHM of $60 \mathrm{~nm}$ ), Red (from 580 to $760 \mathrm{~nm}$, FWHM of $56 \mathrm{~nm}$ ), and IR (700 to $1200 \mathrm{~nm}$, FWHM of $240 \mathrm{~nm}$ ). All photodiodes are covered by a $1 \mathrm{~mm}$ thick PTFE diffuser. Figure 3 presents the relative spectral sensitivity of the combination of each photodiode with the diffuser. The photodiode sensitivities were taken from the manufactures' technical datasheets (Roithner GUVA-S10GD, Hamamatsu S9032-2 and Agilent HSDL-5400). The transmission of the diffuser was measured under stable daylight conditions with a JETI尺 1211UV spectrometer (250-1000 nm range). The 1000-1200 $\mathrm{nm}$ transmission values were extrapolated by using a second degree polynomial fitting the 650$1000 \mathrm{~nm}$ measured values.

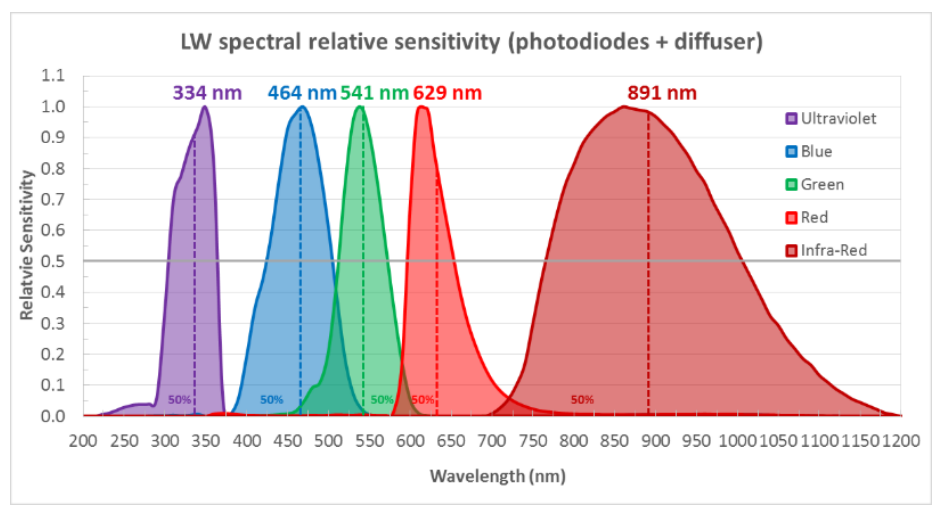

Figure 3 - Relative spectral sensitivity $S_{k}(\lambda)$ of the five photodiodes combined with the diffuser

From each photodiode current, the LW produces a spectral irradiance based on equation 1:

$$
I_{k}=a_{k}+b_{k} \frac{\int_{0}^{\infty} E(\lambda) \cdot S_{k}(\lambda) \cdot d \lambda}{\int_{0}^{\infty} S_{k}(\lambda) \cdot d \lambda} \text { in W.m. } \mathrm{m}^{-2} \cdot \mathrm{nm}^{-1}
$$

where

$E(\lambda)$ is the spectral irradiance on the diffuser in.$W \cdot \mathrm{m}^{-2} \cdot \mathrm{m}^{-1}$;

$S_{k}(\lambda)$ is the relative spectral sensitivity of photodiode+diffuser k (UV, B, G, R or IR);

$a_{k} \quad$ is a calibration coefficient for the photodiode+diffuser $\mathrm{k}$ (dark signal) 
$b_{k} \quad$ is a calibration coefficient for the photodiode+diffuser $\mathrm{k}$

These spectral irradiances are considered to be located at the wavelength which leads to the $50 \%$ value of the integral of the sensitivity curve. Therefore, the UV spectral irradiance is located at $334 \mathrm{~nm}$, the blue one at $464 \mathrm{~nm}$, the green one at $541 \mathrm{~nm}$, the red one at $629 \mathrm{~nm}$ and the IR one at $891 \mathrm{~nm}$. This is illustrated in figure 3. The LW outputs spectral irradiances in $\mathrm{mW} \cdot \mathrm{m}^{-2} \cdot \mathrm{nm}^{-1}$, this is well suited to their order of magnitude under indoor and outdoor conditions.

The LW had been calibrated by the "Object-Tracker" company at the time of the European EUClock project. Before using the device, we checked this calibration with measurements from the JETI® 1211UV spectrometer. This was done under incandescent light (871 and 1423 lux) and daylight (every 5000 lux, from 10000 to 40000 lux). The LW light sensor amplifier factor was set to 8 , a value well suited to indoor and outdoor lighting conditions. The dark signal of each LW photodiode was updated at the same time. Since the $1211 \mathrm{UV}$ did not cover the range $1000 \mathrm{~nm}$ to $1200 \mathrm{~nm}$, Planck's law was used to extend to $1200 \mathrm{~nm}$ the spectra measured under incandescent light and the SMARTS atmospheric radiative transfer model (Gueymard, 1995) was used to extend to $1200 \mathrm{~nm}$ the spectra measured under daylight. The measured spectral irradiances were combined with LW sensitivities to produce 5 spectral irradiances equivalent to the LW spectral irradiances. Figure 4 shows an example for 3 daylight conditions.

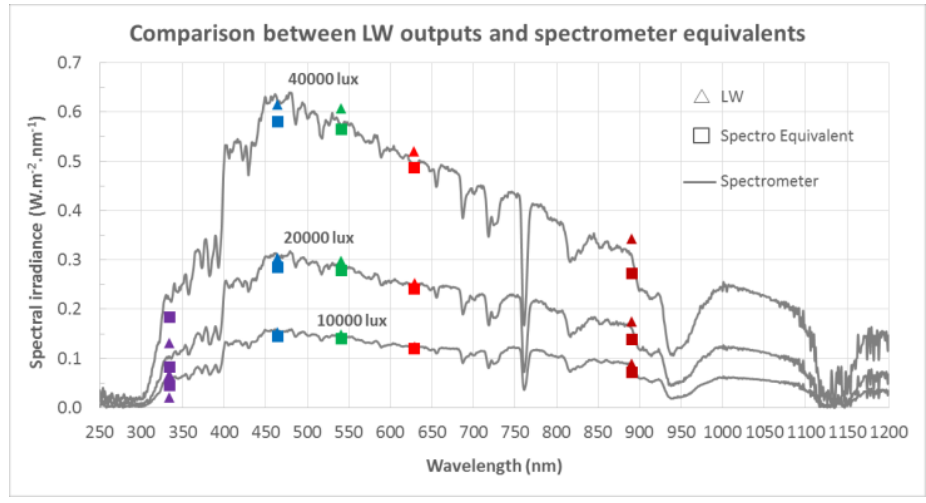

Figure 4 - Comparison between the LW output and 1211 UV spectrometer equivalents

The results of figure 4 are representative of those obtained under all lighting conditions: the UV irradiance was underestimated by the LW; the Blue, Green and Red spectral irradiances were overestimated by the LW, as was the IR spectral irradiance. As a consequence, we decided to apply correction factors to the LW spectral irradiances; these can be found in Table 1 below.

Table 1 - LW spectral irradiance correction factors

\begin{tabular}{|l|l|l|l|l|l|}
\hline & Ultraviolet & Blue & Green & Red & Infrared \\
\hline Multiplier & 1,370 & 0,940 & 0,930 & 0,940 & 0,795 \\
\hline
\end{tabular}

The EUClock version of the LW (type 3.x) does not include photodiodes which can measure directly a photopic illuminance and/or a melanopic equivalent illuminance. Since 2016, a new version is available (type 5.x) which at least includes a photodiode with the $\operatorname{CIE~} V(\lambda)$ sensitivity (Hamamatsu S7686). In order to produce these two illuminances from our LW, we computed a best linear fit approximation of the $V(\lambda)$ and $S_{\text {mel }}(\lambda)(C I E, 2018)$ from the Blue, Green and Red photodiode and diffuser spectral sensitivities. This lead to equation 2 (photopic illuminance) and equation 3 (melanopic equivalent illuminance).

$$
\begin{aligned}
& E_{v}=1.927 \times I_{\text {blue }}+50.315 \times I_{\text {green }}+18.553 \times I_{\text {red }} \text { in lux } \\
& E_{v, \text { mel }}=46.680 \times I_{\text {blue }}+18.223 \times I_{\text {green }}-0.292 \times I_{\text {red }} \text { in melanopic lux }
\end{aligned}
$$

where

Iblue is the spectral irradiance from LW blue photodiode in $\mathrm{mW} \cdot \mathrm{m}^{-2} \cdot \mathrm{nm}^{-1}$ 
$I_{\text {green }}$ is the spectral irradiance from LW green photodiode in $\mathrm{mW} \cdot \mathrm{m}^{-2} \cdot \mathrm{nm}^{-1}$

Ired is the spectral irradiance from LW red photodiode in $\mathrm{mW} \cdot \mathrm{m}^{-2} \cdot \mathrm{nm}^{-1}$

As recommended by ISO and $\mathrm{CIE}(\mathrm{CIE}, 2014)$, we computed the general $\mathrm{V}(\lambda)$ mismatch index $f^{\prime}$ ' using the LW approximation, we found a value of 0.38 . We also applied the mismatch index formula to $S_{\text {mel }}(\lambda)$ using the LW approximation and found a value of 0.39 . A representative value of $f^{\prime}{ }_{1}$ for commercial illuminance meters is 0.02 . This means that the Blue, Green and Red photodiodes used by the LW can only lead to a crude approximation of $V(\lambda)$ or $S_{\text {mel }}(\lambda)$. However, to be reassuring, LW photopic illuminances computed for CIE illuminants D65 (daylight) and A (incandescent) are only $5 \%$ below the $V(\lambda)$ ones while LW melanopic equivalent illuminances for $D 65$ and $A$ are only $5 \%$ above the $S_{\text {mel }}(\lambda)$ ones. The same differences were obtained with the daylight spectra measured on-site when checking the LW calibration.

\subsubsection{Temperature and activity measurements}

The LW also measures temperature with a thermistor, and activity with a 3 axis accelerometer.

As for the photodiodes, the temperature sensor had been calibrated at the time of the European EUClock project. We checked this calibration by comparing the LW to a HOBO U12-013 (Onset, MA, USA) data logger with a built-in temperature sensor. The LW and the HOBO were placed side by side in an environment with a temperature varying from $-20^{\circ} \mathrm{C}$ to $30^{\circ} \mathrm{C}$. Results from the comparison show that for this range, the LW is in line with the HOBO: the mean absolute difference is $-0.4^{\circ} \mathrm{C}$ and the standard deviation is $1.5^{\circ} \mathrm{C}$. However, the $\mathrm{LW}$ is influenced by the body. When it is worn on the eyeglass frame, only $1.5 \mathrm{~cm}$ from the head, its temperature is typically 2 to $3^{\circ} \mathrm{C}$ above the ambient temperature. When it is worn on the wrist, its metallic case is separated from the skin only by the wrist band, its temperature is then well above ambient temperature and close to the skin temperature (around $32^{\circ} \mathrm{C}$ ). It cannot be considered as the skin temperature, since the LW thermistor is not in direct contact with the skin.

The 3 axis accelerometer chip and the LW electronic board are positioned inside the case so that each axis is parallel to one dimension of the case. When worn on the eyeglass frame, the $X$ axis is the horizontal (the length), the $Y$ axis is the vertical (the height) and $Z$ (the width) is perpendicular to both. We added marks on the outside of the case to make sure that the LW was always positioned the same way ( $\mathrm{Y}$ axis up). The measuring range is $\pm 3 \mathrm{~g}$ with a resolution of $0.002 \mathrm{~g}$. For the analysis, we used the 3 accelerometer measurements to produce an activity index which expresses their time variations, it is defined by equation 4 :

$$
I_{a c t}=\frac{\sqrt{[x(t)-x(t-1)]^{2}+[y(t)-y(t-1)]^{2}+[z(t)-z(t-1)]^{2}}}{3} \text { in g }
$$

\subsubsection{Measurement mode}

Taking into account the LW memory $(4 \mathrm{Mb})$ and the battery capacity $(100 \mathrm{mAh})$, we chose to measure light, activity and temperature every 10 seconds. This gave us an autonomy of about 6 days. The light sensor amplifier was set to a factor of 8 . As explained before, the LW was worn on an eyeglass frame during the day (always on the same side). During bedtime, it was worn on the wrist, the diffuser window facing toward the hand in order to be able to measure light exposure during the night. The LW was not worn during showers. Every 5 days, we connected the LW to a computer to transfer the data collected and charge its battery. This took about 1 hour and a half. The LW could not be worn at the same time so this was done in the evening while watching TV, a time of low exposure to light and little activity.

\section{Data processing and exploratory analysis}

\subsection{Quality control and activity log adjustment}

A few days after collecting the data measured by the LW, the 10 s measurements and the activities recorded in the log were plotted together day by day. Figure 5 (light measurements) and figure 6 (accelerometer and temperature) give an example for a working day in June. Light measurements are presented in $\mathrm{mW} \cdot \mathrm{m}^{-2} \cdot \mathrm{nm}^{-1}$ using a $\log _{10}$ scale. A 24 hour time scale is used and dark areas indicate night-time. In both figures, the last graph indicate the activities recorded in the log during the day. These plots were routinely used to (1) visually control the 
measurements and (2) adjust the time of the activities by a few minutes when the measurements clearly provided more precise information.

Figure 5 corresponds to a working day. A bike was used to commute to work: the exposure to outdoor daylight is marked by IR, R, G, B irradiance levels above $40 \mathrm{~mW} \cdot \mathrm{m}^{-2} \cdot \mathrm{nm}^{-1}$ and UV is present. The morning was spent at the office ( $1 \mathrm{~m}$ from a window facing west), the afternoon was spent in a meeting room (in the middle of east and west facing windows). The exposure to indoor daylight is marked by IR, R, G, B irradiance levels below $10 \mathrm{~mW} \cdot \mathrm{m}^{-2} \cdot \mathrm{nm}^{-1}$, UV has been filtered by the window glass. After 22:00, just before going to bed, notice the predominant IR and $\mathrm{R}$ irradiances marking the use of an incandescent light in the bathroom.

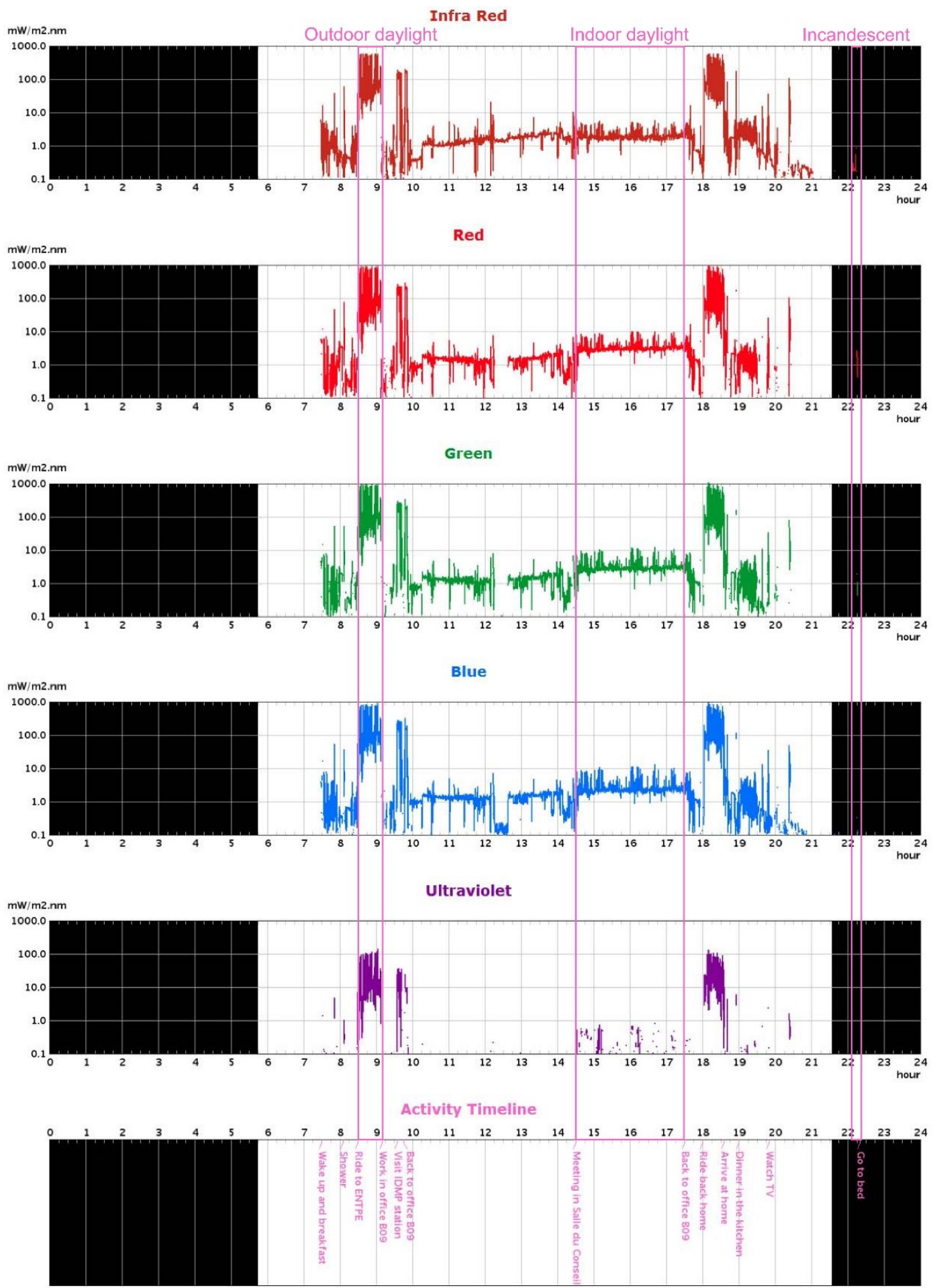

Figure 5 - One day of LW light measurements with activities 
Figure 6 presents the accelerometer and the temperature measurements for the same working day as figure 5 . The $\mathrm{LW}$ is worn on the wrist during bedtime then on the eyeglass frame. This change is marked by the $90^{\circ}$ rotation of the $Y$ and $Z$ axis ( $Y$ goes from 0 to 1 and $Z$ from 1 to 0 ) and by a decrease in temperature: from $32^{\circ} \mathrm{C}$ to $24^{\circ} \mathrm{C}$. Notice the difference in the accelerometer variations between sleep and awake periods. There are also differences between working on the computer in the morning and taking part to a meeting in the afternoon (increased head movements). Notice also the steady temperature increase in the working office (west oriented).

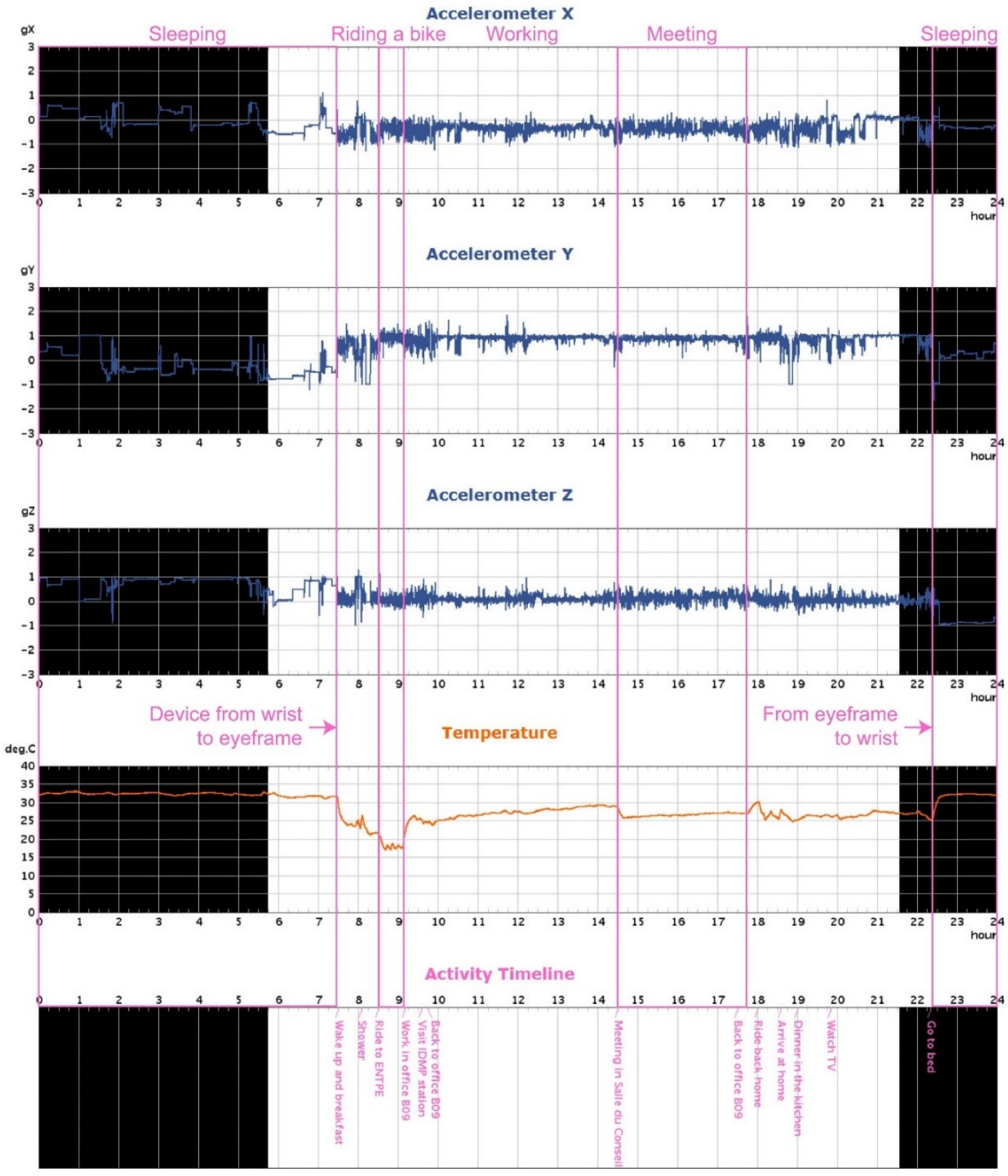

Figure 6 - One day of LW accelerometer and temperature measurements with activities

\subsection{Data processing}

The acquisition started on January 7, 2014 and ended on January 11, 2015. For the sake of presentation, January 1 to 6,2015 were used as a substitute for January 1 to 6,2014 . A full day of data (24 hours, every 10 seconds) accounts for 8640 recordings. Every 5 days, the acquisition had to be stopped in the evening to charge the LW and save the data. For these days, we miss from 1 to 2 hours. On 5 days (January 23, May 30 and 31, June 1 and December 21 ) the acquisition stopped because the battery had not been charged long enough. Figure 7 shows day by day, for the entire year, the number of hours with missing measurements. 


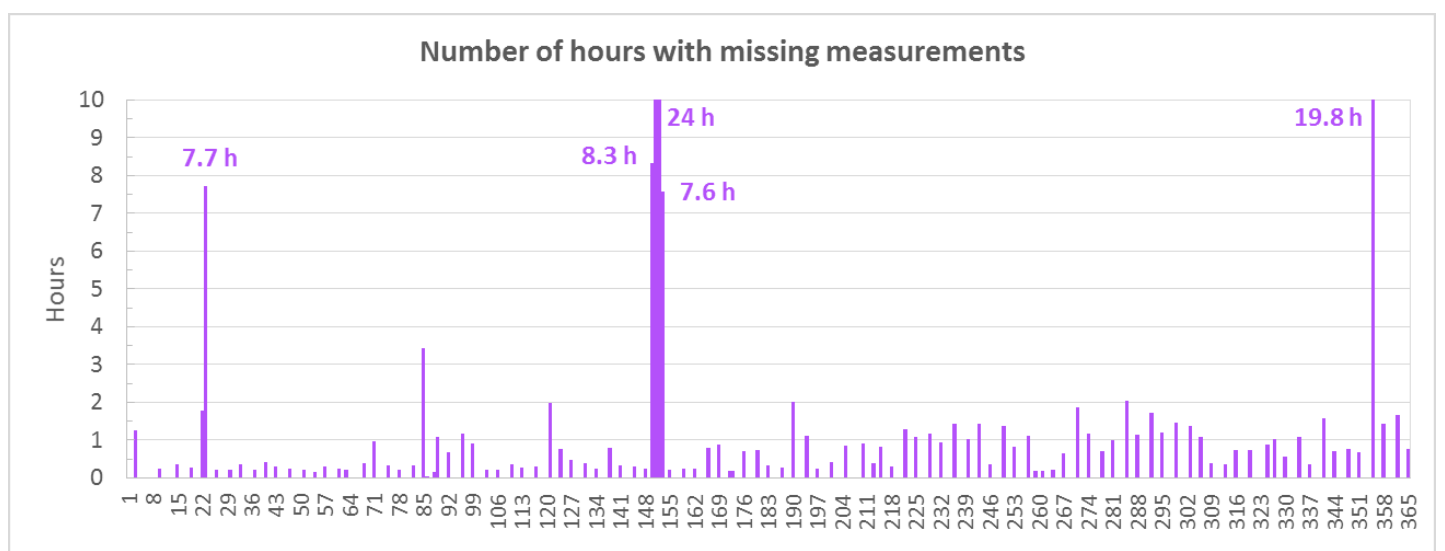

Julian day

Figure 7 - Number of hours with missing measurements for each day of the year

We used the 10 s measurements available to compute the illuminance (equation 2) and the melanopic equivalent illuminance (equation 3 ) as well as the activity index (equation 4). From the 10 s measurements, we computed one minute averages. Minutes with 10 s measurements missing were considered as missing (values set to -99). Our exposure to light depends on our activities and on our outdoor environment. Therefore, to prepare the analysis, we flagged every minute of each day with indicators of: (1) the day of the week (1=Monday, $7=$ Sunday), (2) whether this was a working day $(0=$ no, $1=y e s)$ and ( 3$)$ whether the minute was within daytime $(0=$ no, $1=y e s)$. Finally, to visualize in one shot, the annual variations of all parameters measured or computed, we designed a plot (figure 8 ) where pixels are colored according to the value of the minute data and placed according to the day of the year $(x)$ and the minute of the day $(y)$.

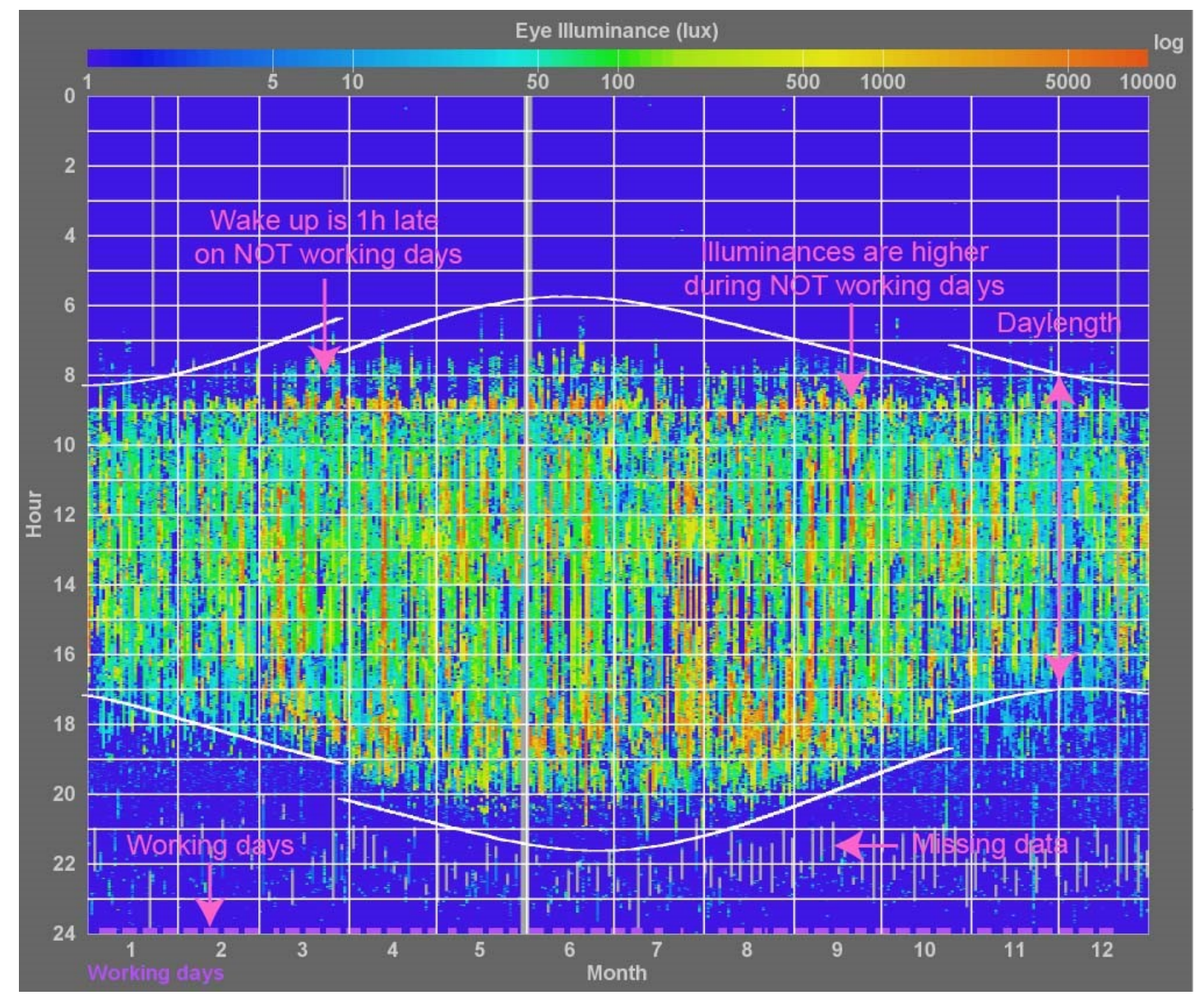

Figure 8 - Minute values of eye illuminance throughout the year. 
Figure 8 shows the minute variations of the eye illuminance throughout the year, the scale is logarithmic and covers the range 1 to 10000 lux (even though the max illuminance is 62000 lux only $1,2 \%$ of the values are above 10000 lux). Vertical grey bars indicate days and hours when measurements were missing. As mentioned before, this happens when the LW was being recharged, i.e. while watching TV, usually after 21:00. The hours are expressed using clock time (GMT+1 and DST/GMT+2 from March 30 to October 25). The two white lines in the early morning and the late evening give the daytime limits throughout the year. Finally, just above the month axis, there is an indication of working days using a short line drawn in purple.

Figure 8 shows clearly the difference in behaviour between working days and NOT working days (weekend or vacation). On "NOT working" days, wake up is one hour late and illuminance levels are higher due to outdoor activities. Table 2 shows how minute values of the illuminance are distributed throughout the year: $58 \%$ of the lit situations (>1lux) correspond to eye illuminances below 100 lux, $12.7 \%$ correspond to bright light situations (>1000 Ix). "Working days" contribute to more than $60 \%$ of the two lowest range of illuminance. On the opposite, "NOT working" days contribute to more than $60 \%$ of the two highest.

Table 2 - Distribution of minute illuminance values - NOT Working and Working days

\begin{tabular}{|l|l|l|l|l|}
\hline $\begin{array}{l}\text { Illuminance Range } \\
(\text { lux })\end{array}$ & $\begin{array}{l}\text { All days } \\
\text { (hours) }\end{array}$ & $\begin{array}{l}\text { All days (\% of } \\
\text { total }>1 \text { lux) }\end{array}$ & $\begin{array}{l}\text { NOT working } \\
\text { days (\% for } \\
\text { the range) }\end{array}$ & $\begin{array}{l}\text { Working } \\
\text { days (\% for } \\
\text { the range) }\end{array}$ \\
\hline $1<\mathrm{E}_{\mathrm{v}} \leq 100 \mathrm{Ix}$ & $2208,4 \mathrm{~h}$ & $58,7 \%$ & $29,4 \%$ & $70,6 \%$ \\
\hline $100<\mathrm{E}_{\mathrm{v}} \leq 1000 \mathrm{Ix}$ & $955,6 \mathrm{~h}$ & $25,4 \%$ & $39,5 \%$ & $60,5 \%$ \\
\hline $1000<\mathrm{E}_{\mathrm{v}} \leq 10000 \mathrm{Ix}$ & $433,2 \mathrm{~h}$ & $11,5 \%$ & $65,1 \%$ & $34,9 \%$ \\
\hline $10000<\mathrm{E}_{\mathrm{v}} \leq 100000 \mathrm{Ix}$ & $43,8 \mathrm{~h}$ & $1,2 \%$ & $61,9 \%$ & $38,1 \%$ \\
\hline
\end{tabular}

\section{Conclusion and perspectives}

Wearing the "LightWatcher" (LW) during an entire year allowed us to test the routine use of a light dosimeter and to gather a unique dataset. Of course, the data is only representative of one person behaviour, this will prevent any trend to be drawn from its analysis. However, it is a good starting point to prepare future studies involving larger panels. To help in developing new and well suited analysis methods, the data collected will be made available to other researchers via a data sharing platform.

Regarding the LW light measurements, we find that UV is useful because it is a marker of outdoor situations and it can cause eye damage and skin burns. Measuring UV, B, G, R and IR gives spectral information which can potentially be used to derive the type of light source(s), to compute illuminances and provide information on the excitation level of the various photoreceptors. We have seen however that combining the $B, G$ and $R$ signals from the LW photodiodes could provide only crude approximations to $V(\lambda)$ or $S_{\text {mel }}(\lambda)$. This is the reason why since 2016, a new version of the LW (type 5.x) includes a photodiode with the CIE $V(\lambda)$ sensitivity. However, this is not enough, we definitely need more detailed information on the light spectrum; multispectral sensor chips are becoming available and new devices should rely on them to provide improved spectral information.

The LW is more than a light dosimeter since it also records activity and temperature. Actimetry is essential to estimate the activity level during the day as well as the quality of sleep during the night. During the day, the LW measures activity from the head movements rather than the wrist movements, which is the usual way. We should investigate what difference that makes. However, we find advantages to it, like knowing the head tilt angle and identifying bad postures leading to neck pain. LW temperatures are useful because they complement the information brought by light measurements on the type of environments in which we live throughout the day. During the night, measuring directly skin temperature could complement actimetry to monitor sleep quality, however this would require to position the LW thermistor on the metallic case and find a wrist band which does not prevent the LW case to touch the skin. 
Regarding its practical use, the dosimeter was light enough $(\sim 15 \mathrm{~g})$, so that at the end of the day, we were not tired of wearing it on the eyeglass frame. During the first weeks, we were wondering whether it would attract the attention of people at work, in the street, in shops... Not much in fact, since people are used to Bluetooth headsets which have about the same size and are also worn close to the ear. However, we changed its colour from white to black. What was really cumbersome was the need for charging the LW and saving its data every 5 days (73 times in a year). There would have been a real danger to loose data with subjects far less concerned by the study than we were. To improve this, the LW autonomy (battery and data storage) should be increased to at least a month.

Finally, we forced ourselves in filling everyday an activity log, this was required for the analysis of the measurements, but this was a real burden. In the future, light spectrum, activity, and temperature combined with GPS data could automatically provide this information using machine learning methods. It would only need to be validated by the subjects once a week.

\section{References}

Auger, R.R., Burgess, H.J., Emens, J.S., Deriy, L.V., Thomas, S.M., Sharkey, K.M., 2015. Clinical Practice Guideline for the Treatment of Intrinsic Circadian Rhythm Sleep-Wake Disorders. Journal of Clinical Sleep Medicine 11, 1199-1236. https://doi.org/10.5664/jcsm.5100

Bierman, A., Klein, T.R., Rea, M.S., 2005. The Daysimeter: a device for measuring optical radiation as a stimulus for the human circadian system. Measurement Science and Technology 16, 2292-2299. https://doi.org/10.1088/0957-0233/16/11/023

Brainard, G.C., Hanifin, J.P., Greeson, J.M., Byrne, B., Glickman, G., Gerner, E., Rollag, M.D., 2001. Action spectrum for melatonin regulation in humans: evidence for a novel circadian photoreceptor. Journal of Neuroscience 21, 6405-6412.

Chang, A.-M., Aeschbach, D., Duffy, J.F., Czeisler, C.A., 2015. Evening use of light-emitting eReaders negatively affects sleep, circadian timing, and next-morning alertness. Proceedings of the National Academy of Sciences 112, 1232-1237. https://doi.org/10.1073/pnas.1418490112

CIE, 2018. CIE/S026 E:2018 CIE System for Metrology of Optical Radiation for ipRGCInfluenced Responses to Light. Vienna: CIE.

CIE, 2014. ISO/CIE 19476:2014 Characterization of the performance of illuminance meters and luminance meters.

CIE, 2001. CIE-139 The influence of daylight and artificial light on diurnal and seasonal variations in humans: a bibliography. International Commission on Illumination, Vienna, Austria.

Cole, R.J., Kripke, D.F., Wisbey, J., Mason, W.J., Gruen, W., Hauri, P.J., Juarez, S., 1995. Seasonal Variation in Human Illumination Exposure at Two Different Latitudes. J Biol Rhythms 10, 324-334. https://doi.org/10.1177/074873049501000406

Czeisler, C.A., 2013. Perspective: Casting light on sleep deficiency. Nature 497, S13-S13. https://doi.org/10.1038/497S13a

Eastman, C.I., 1990. Natural summer and winter sunlight exposure patterns in seasonal affective disorder. Physiol. Behav. 48, 611-616.

Gueymard, C., 1995. SMARTS2: A Simple Model of the Atmospheric Radiative Transfer of Sunshine: algorithms and performance assessment. (No. FSEC-PF-270-95). Florida Solar Energy Center, Cocoa, Florida.

Hubalek, S., 2007. LuxBlick: Messung der täglichen Lichtexposition zur Beurteilung der nichtvisuellen Lichtwirkungen über das Auge. ETH Zurich. https://doi.org/10.3929/ethz-a005429531

Lucas, R.J., Peirson, S.N., Berson, D.M., Brown, T.M., Cooper, H.M., Czeisler, C.A., Figueiro, M.G., Gamlin, P.D., Lockley, S.W., O’Hagan, J.B., Price, L.L.A., Provencio, I., Skene, D.J., 
Brainard, G.C., 2014. Measuring and using light in the melanopsin age. Trends in Neurosciences 37, 1-9. https://doi.org/10.1016/j.tins.2013.10.004

OT, 2016. LightWatcher - Personal Data Recorder - Manual (No. Version 3.16). Wolf Technologie - Object Tracker.

Scheuermaier, K., Laffan, A.M., Duffy, J.F., 2010. Light Exposure Patterns in Healthy Older and Young Adults. J Biol Rhythms 25, 113-122. https://doi.org/10.1177/0748730410361916 